\title{
Longitudinal pathways of exclusive and polytobacco cigarette use among youth, young adults and adults in the USA: findings from the PATH Study Waves 1-3 (2013-2016)
}

\author{
Kristie A Taylor 이, ' Eva Sharma 다, ' Kathryn C Edwards (1) , \\ Michael J Halenar (1) , 'Wendy Kissin, ${ }^{1}$ Karin A Kasza (1) , ${ }^{2}$ Hannah Day, ${ }^{3}$ \\ Gabriella Anic, ${ }^{3}$ Lisa D Gardner (1), ${ }^{3}$ Hoda T Hammad, ${ }^{3}$ Lynn C Hull, ${ }^{3}$ \\ Maansi Bansal-Travers (1) , ${ }^{2}$ Jean Limpert, ${ }^{3}$ Nicolette Borek, ${ }^{3}$ Heather L Kimmel (1) , ${ }^{4}$ \\ Wilson M Compton, ${ }^{4}$ Andrew Hyland, ${ }^{2}$ Cassandra Stanton (1) ${ }^{1,5}$
}

\begin{abstract}
- Additional material is published online only. To view please visit the journal online (http://dx.doi.org/10.1136/ tobaccocontrol-2020-055630).
\end{abstract}

${ }^{1}$ Behavioral Health and Health Policy Practice, Westat, Rockville, MD, United States

'Department of Health Behavior, Roswell Park Comprehensive Cancer Center, Buffalo, NY, United States

${ }^{3}$ Office of Science, Center for Tobacco Products, Food and Drug Administration, Silver Spring, MD, United States ${ }^{4}$ National Institute on Drug Abuse, National Institutes of Health, Bethesda, MD, United States

${ }^{5}$ Department of Oncology, Georgetown University Medical Center, Washington, DC, United States

\section{Correspondence to}

Dr Kristie A Taylor, Behavioral Health and Health Policy, Westat, Rockville, Maryland, USA; KristieTaylor@westat.com

Received 15 January 2020 Revised 22 January 2020 Accepted 27 January 2020
Check for updates

(C) Author(s) (or their employer(s)) 2020. No commercial re-use. See rights and permissions. Published by BMJ.

To cite: Taylor KA, Sharma $E_{\text {, }}$ Edwards $\mathrm{KC}$, et al.

Tob Control

2020;29:s139-s146.

\section{ABSTRACT}

Objective Cigarettes are the most harmful and most prevalent tobacco product in the USA. This study examines cross-sectional prevalence and longitudinal pathways of cigarette use among US youth (12-17 years), young adults (18-24 years) and adults $25+(25$ years and older).

Design Data were drawn from the first three waves (2013-2016) of the Population Assessment of Tobacco and Health Study, a nationally representative, longitudinal cohort study of US adults and youth. Respondents with data at all three waves (youth, $\mathrm{N}=11$ 046; young adults, $\mathrm{N}=6478$; adults $25+, \mathrm{N}=17$ 188) were included in longitudinal analyses.

Results Among Wave 1 (W1) any past 30-day (P30D) cigarette users, more than $60 \%$, persistently used cigarettes across three waves in all age groups. Exclusive cigarette use was more common among adult 25+ W1 P30D cigarette users (62.6\%), while cigarette polytobacco use was more common among youth $(57.1 \%)$ and young adults (65.2\%). Persistent exclusive cigarette use was the most common pathway among adults $25+$ and young adults; transitioning from exclusive cigarette use to cigarette polytobacco use was most common among youth W1 exclusive cigarette users. For W1 youth and young adult cigarette polytobacco users, the most common pattern of use was persistent cigarette polytobacco use.

Conclusions Cigarette use remains persistent across time, regardless of age, with most W1 P30D smokers continuing to smoke at all three waves. Policy efforts need to continue focusing on cigarettes, in addition to products such as electronic nicotine delivery systems that are becoming more prevalent.

\section{INTRODUCTION}

Cigarettes are the most common tobacco product in the USA, with approximately 61 million people in 2016 having smoked cigarettes in the past year, almost 2 million of them younger than age $18 .^{1}$ Cigarette use is more than twice as prevalent as the use of any other tobacco product among US adults; in 2014, 18.1\% currently smoked cigarettes, and $16.0 \%$ smoked cigarettes daily. ${ }^{2}$ Furthermore, cigarettes are the product most commonly used by tobacco users, including those who use one product and those who use multiple products. ${ }^{2-4}$

Tobacco products have been conceptualised as falling along a continuum of risk, ${ }^{56}$ with cigarettes on the most harmful end of the continuum considering exposure to harmful chemicals multiplied by vast number of users. ${ }^{7}$ Although cigarette smoking prevalence has decreased over the past decades, ${ }^{8-10}$ the resulting public health benefit may be tempered by evolving patterns of cigarette use, including increases in non-daily cigarette smoking, ${ }^{10}$ and disparities in cigarette smoking prevalence based on race/ethnicity, ${ }^{11}{ }^{12}$ socioeconomic status, ${ }^{13}{ }^{14}$ sexual orientation and/or gender identity. ${ }^{1516}$

Polytobacco use, or use of more than one tobacco product, is increasing ${ }^{10}$ and is associated with continued cigarette smoking behaviour and nicotine dependence among youth and adults. ${ }^{17-19}$ Among youth and adults, approximately 40\% of tobacco users use multiple tobacco products, ${ }^{2}$ with cigarette polytobacco use (using cigarettes plus at least one other product) consistently included in the top combinations of products. ${ }^{242-22}$ Among adult smokers, $16.3 \%$ smoked cigarettes with at least one other tobacco product, ${ }^{23}$ while among young adult smokers, $22.6 \%$ were polytobacco users ${ }^{23}$ and among youth cigarette smokers in grades $6-12$, $46.1 \%$ smoked cigarettes and also used one or more other tobacco products. $^{24}$

This study draws from the longitudinal cohort design of the Population Assessment of Tobacco and Health (PATH) Study and examines pathways of cigarette use in the USA across three waves from 2013 to 2016 . We first provide overall crosssectional weighted estimates of ever: past 12-month (P12M), past 30-day (P30D) and daily P30D use for US youth (aged 12-17), young adults (aged 18-24) and adults aged 25 and older (adults 25+) from 2013 to 2016. Using the first three waves of longitudinal within-person data from the PATH Study, Aim 2 is to examine whether the known age group differences in tobacco use discussed above are found among the pathways of persistent use, discontinued use and reuptake of cigarettes among Wave 1 (W1) P30D cigarette users. The focus is 
on P30D use to provide a broad overview of the transitions in cigarette use. Aim 3 is to compare longitudinal transitions of use among W1 exclusive cigarette smokers and W1 cigarette smokers who use multiple tobacco products (cigarette polytobacco use) to understand broad transitions such as tobacco cessation, tobacco reuptake, persistent cigarette use, discontinued cigarette use and switching to another tobacco product. Monitoring these longitudinal transitions among cigarette smokers separately for exclusive and polytobacco users will advance our understanding of critical product transitions, improving comprehension of the health risks associated with cigarette smoking at the population level. The important topic of initiation of cigarette use is addressed in another paper in this supplement issue. ${ }^{25}$

\section{METHODS}

\section{Study design and population}

The PATH Study is an ongoing, nationally representative, longitudinal cohort study of youth (aged 12-17) and adults (aged 18 or older) in the US. Self-reported data were collected using audio computer-assisted self-interviews administered in English and Spanish. Further details regarding the PATH Study design and W1 methods are published elsewhere. ${ }^{26}{ }^{27}$ At W1, the weighted response rate for the household screener was 54.0\%. Among screened households, the overall weighted response rate was $78.4 \%$ for youth and $74.0 \%$ for adults at W1, $87.3 \%$ for youth and $83.2 \%$ for adults at Wave 2 (W2) and $83.3 \%$ for youth and $78.4 \%$ for adults at Wave 3 (W3). Details on interview procedures, questionnaires, sampling, weighting and adjustments for non-response, and information on accessing the data are available online (https://doi.org/10.3886/Series606). The study was conducted by Westat and approved by the Westat Institutional Review Board. All participants aged 18 and older provided informed consent, with youth participants aged $12-17$ providing assent while their parent/legal guardian provided consent.

This study reports cross-sectional estimates from 13651 youth and 32320 adults who participated in W1 (data collected from 12 September 2013 through 14 December 2014), 12172 youth and 28362 adults at W2 (data collected from 23 October 2014 through 30 October 2015) and 11814 youth and 28148 adults at W3 (data collected from 19 October 2015 to 23 October 2016). The differences in the number of completed interviews between W1, W2 and W3 reflect attrition due to non-response, mortality and other factors, as well as youth who enrolled in the study at W2 or W3. ${ }^{26}$ We also report longitudinal estimates from W1 youth $(n=11046)$, W1 young adults $(n=6478)$ and $W 1$ adults $25+(n=17188)$ with data collected at all three waves. See online supplementary figure 1 for a detailed description of the analytic sample for longitudinal analysis.

\section{Measures}

\section{Tobacco use}

At each wave, adults and youth were asked about their tobacco use behaviours for cigarettes and electronic nicotine delivery systems, including e-cigarettes, traditional cigars, cigarillos, filtered cigars, pipe tobacco, hookah, snus pouches, other smokeless tobacco and dissolvable tobacco. Participants were asked about P30D use of 'e-cigarettes' at W1 and W2 and 'e-products' (e-cigarettes, e-cigars, e-pipes and e-hookah) at W3; all electronic products noted above are referred to as ENDS. In addition, youth were asked about their use of bidis and kreteks. However, use of bidis, kreteks and dissolvable tobacco were not included in the analyses due to small sample sizes.

\section{Outcome measures}

Cross-sectional definitions of use included ever, P12M, P30D and daily P30D use. Longitudinal outcomes included persistent cigarette use, discontinued cigarette use and reuptake of cigarette use, as well as transitions among exclusive and polytobacco cigarette users. The definition of each outcome is included in the footnote of the table/figure in which it is presented.

\section{Analytic approach}

To address Aim 1, weighted cross-sectional prevalence of cigarette use was estimated across ever, P12M, P30D and daily P30D use at each wave stratified by age. For Aim 2, irrespective of other tobacco product use, longitudinal W1-W2-W3 transitions in P30D cigarette use were summarised to represent pathways of persistent any P30D cigarette use (defined as continued exclusive or polytobacco cigarette use at W2 and W3), discontinued any P30D cigarette use (stopped cigarette use at W2 and W3 or just W3) and reuptake of any P30D cigarette use (used cigarettes at W1, discontinued cigarette use at W2 and used cigarettes again at W3). Finally, to address Aim 3, longitudinal W1-W2W3 cigarette use pathways that flow through five mutually exclusive and exhaustive transition categories were examined for W1 P30D exclusive cigarette use and W1 P30D cigarette polytobacco use (see online supplementary figure 2). These pathways represent building blocks that may be aggregated to reflect higher level behavioural transitions, such as discontinued tobacco use, tobacco use reuptake, persistent use, transition to exclusive or polytobacco use, exclusive use reuptake, switching product use and inconsistent use. For each aim, weighted t-tests were conducted on differences in proportions to assess statistical significance. To correct for multiple comparisons, Bonferroni post hoc tests were used where relevant.

Cross-sectional estimates (Aim 1) were calculated using the PATH Study cross-sectional weights for W1 and single-wave (pseudo-cross-sectional) weights for W2 and W3. The weighting procedures adjusted for complex study design characteristics and non-response. Combined with the use of a probability sample, the weighted data allow these estimates to be representative of the non-institutionalised, civilian, resident US population aged 12 or older at the time of each wave. Longitudinal estimates (Aims 2 and 3) were calculated using the PATH Study W3 allwaves weights. These weighted estimates are representative of the resident US population aged 12 and older at the time of W3 (other than those who were incarcerated) who were in the civilian, non-institutionalised population at W1.

All analyses were conducted using SAS Survey Procedures, V.9.4 (SAS Institute). Variances were estimated using the balanced repeated replication method ${ }^{28}$ with Fay's adjustment set to 0.3 to increase estimate stability. ${ }^{29}$ Analyses were run on the W1-W3 Public Use Files (https://doi.org/10.3886/ICPSR36498.v8). Estimates with low precision (those based on fewer than 50 observations in the denominator or with a relative standard error greater than 0.30 ) were flagged and are not discussed in the Results. Respondents missing a response to a composite variable (eg, ever, P30D) were treated as missing; missing data were handled with listwise deletion.

\section{RESULTS}

\section{Cross-sectional weighted prevalence}

As shown in figure 1 , for youth, there were statistically significant decreases in prevalence of ever use of cigarettes at every wave from $13.4 \%(95 \%$ CI 12.6 to 14.3$)$ at W1, $11.7 \%(95 \% \mathrm{CI}$ 11.0 to 12.3$)$ at W2 to $10.7 \%(95 \%$ CI 10.0 to 11.4$)$ at W3. For 


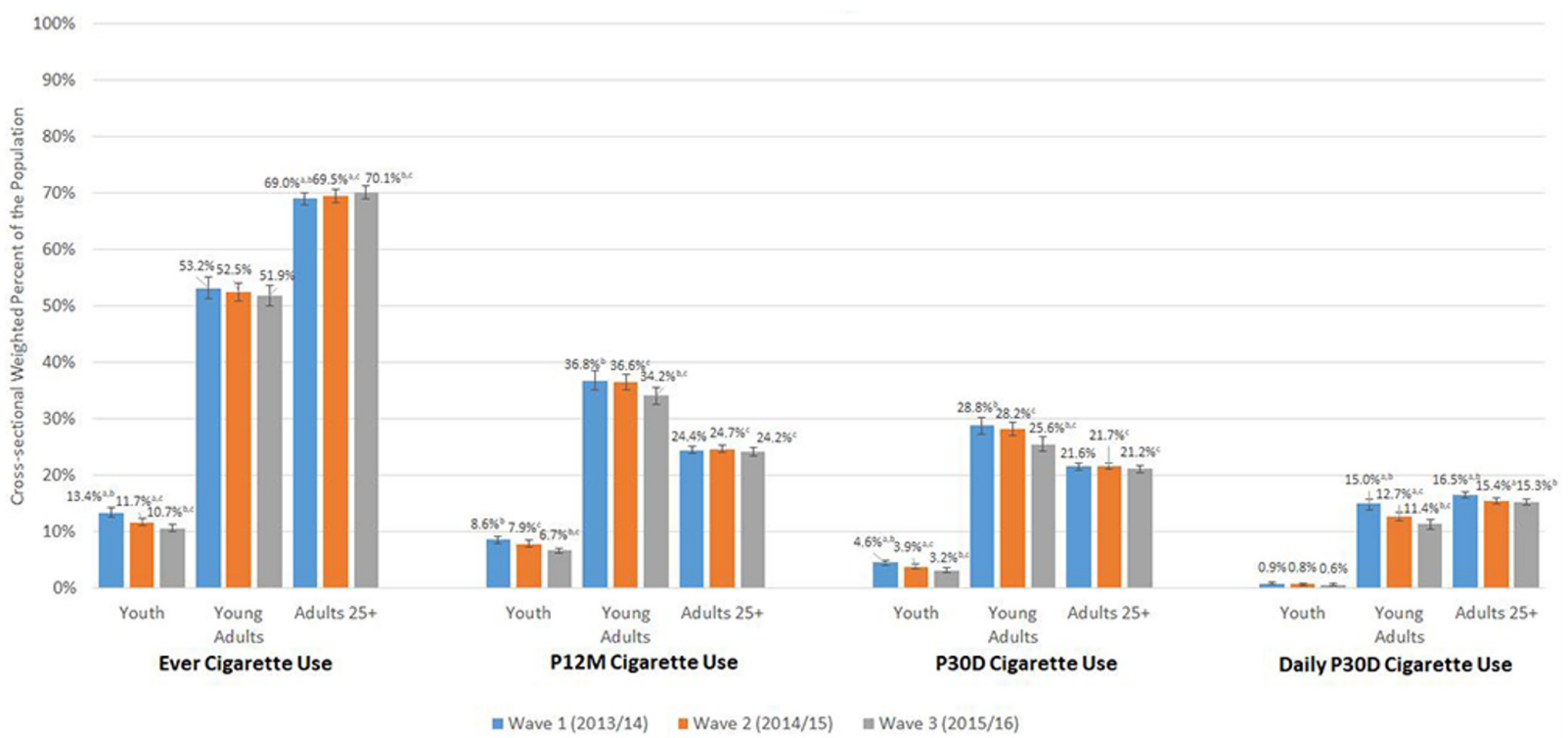

Figure 1 Cross-sectional weighted percent of ever, P12M, P30D and daily P30D cigarette use among youth, young adults and adults 25+ in W1, W2 and W3 of the Population Assessment of Tobacco and Health (PATH) Study. P12M, past12-month; P30D, past30-day; W1, Wave 1; W2, Wave 2; W3, Wave 3.

Abbreviations: P12M = past 12-month; P30D = past 30-day; $\mathrm{W} 1=$ Wave $1 ; \mathrm{W} 2=$ Wave 2; $\mathrm{W} 3=$ Wave 3

W1/W2/W3 ever cigarette use unweighted Ns: youth (ages 12-17) = 1,838/1,428/1,220; young adults (ages 18-24) = 5,963/4,853/4,580; adults 25+ (ages 25 and older) $=19,213 / 16,790 / 16,305$

W1/W2/W3 P12M cigarette use unweighted Ns: youth =1,170/965/757; young adults $=4,392 / 3,564 / 3,202$; adults $25+=11,538 / 9,927 / 9,267$

W1/W2/W3 P30D cigarette use unweighted Ns: youth $=634 / 481 / 366$; young adults $=3,593 / 2,799 / 2,456$; adults $25+=10,624 / 8,880 / 8,275$

W1/W2/W3 daily P30D cigarette use unweighted Ns: youth $=127 / 96 / 73$; young adults $=1,931 / 1,338 / 1,138$; adults $25+=8,236 / 6,596 / 6,229$

$X$-axis shows four categories of cigarette use (ever, P12M, P30D, and daily P30D). Y-axis shows weighted percentages of W1, W2, and W3 users.

Sample analyzed includes all W1, W2, and W3 respondents at each wave. All respondents with data at one wave are included in the sample for that wave's estimate and do not need to have complete data at all three waves. The PATH Study cross-sectional (W1) or single-wave weights (W2 and W3) were used to calculate estimates at each wave. W1-W3 ever cigarette use is defined as having smoked cigarettes, even once or twice in lifetime. W1W3 P12M cigarette use is defined as any cigarette use within the past 12 months. W1-W3 P30D cigarette use is defined as any cigarette use within the past 30 days. W1-W3 daily P30D cigarette use is defined as cigarette use every day within the past 30 days. All use definitions refer to any use that includes exclusive or polytobacco use of cigarettes.

adenotes significant difference at $\mathrm{p}<0.0167$ (Bonferroni corrected for three comparisons) between W1 and W2 bdenotes significant difference at $p<0.0167$ (Bonferroni corrected for three comparisons) between W1 and W3 'denotes significant difference at $\mathrm{p}<0.0167$ (Bonferroni corrected for three comparisons) between W2 and W3 The logit-transformation method was used to calculate the $95 \%$ confidence intervals.

Analyses were run on the W1, W2, and W3 Public Use Files (https://doi.org/10.3886/ICPSR36498.v8).

adults $25+$, there are small increases in prevalence of ever use across waves that are statistically significant: ever use increased from $69.0 \%(95 \%$ CI 67.9 to 70.1$)$ at W1, to $69.5 \%(95 \%$ CI 68.3 to 70.7$)$ at W2 to $70.1 \%(95 \% \mathrm{CI} 68.9$ to 71.3$)$ at W3. For P12M cigarette use, among youth there was a decline between W1 $(8.6 \%$ (95\% CI 8.0 to 9.3$)$ ) and W3 (6.7\% (95\% CI 6.3 to 7.2$)$ ). Similarly, among young adults, there was a decline between W1 (36.8\% (95\% CI 35.2 to 38.5$))$ and W3 (34.2\% (95\% CI 32.7 to 35.6$)$ ). For adults $25+$, there was a statistically significant decline between W2 (24.7\% (95\% CI 24.1 to $25.4)$ ) and W3 (24.2\% (95\% CI 23.4 to 24.9)). Prevalence of P30D cigarette use decreased among youth between W1 (4.6\% (95\% CI 4.2 to 5.0$)$ ) and W3 (3.2\% (95\% CI 2.8 to 3.6)), and among young adults P30D cigarette use decreased between W2 (28.2\% (95\% CI 27.0 to 29.4$))$ and W3 $(25.6 \%$ (95\% CI 24.3 to 26.9)). Among adults $25+$, P30D cigarette use decreased slightly between W2 and W3, from 21.7\% (95\% CI 21.1 to 22.3 ) at W2 to $21.2 \%$ (95\% CI 20.5 to 21.8 ) at W3. Across all three waves, less than $1 \%$ of youth smoked cigarettes every day in the past 30 days. Among young adults, daily use of cigarettes in the past 30 days decreased between W1 (15.0\% (95\% CI 14.0 to 15.9$)$ ), W2
(12.7\% (95\% CI 11.9 to 13.5$))$ and W3 (11.4\% (95\% CI 10.5 to 12.3)). Among adults $25+$, daily use declined between W1 (16.5\% (95\% CI 16.0 to 17.1)) and W2 (15.4\% (95\% CI 14.9 to 16.0$)$ ) and remained about the same at W3.

\section{Longitudinal weighted W1-W2-W3 pathways}

To address Aim 2, figure 2 illustrates the potential longitudinal pathways among those who smoked cigarettes in the P30D at W1.

\section{Among P30D cigarette users at W1}

Among those with data from all waves (W1, W2 and W3), 4.5\% (95\% CI 4.1 to 5.0 ) of youth, $28.3 \%$ (95\% CI 26.8 to 29.7 ) of young adults and $21.3 \%$ (95\% CI 20.7 to 22.0$)$ of adults $25+$ were P30D cigarette users at W1. Persistent P30D cigarette use, regardless of concurrent P30D use of other products, was found among $60.4 \%$ (95\% CI 55.0 to 65.5 ) of youth, $68.1 \%$ (95\% CI 66.0 to 70.2$)$ of young adults and $82.3 \%$ (95\% CI 81.3 to 83.4$)$ of adults $25+$, and the differences among all three age groups were statistically significant. Discontinued cigarette use occurred 


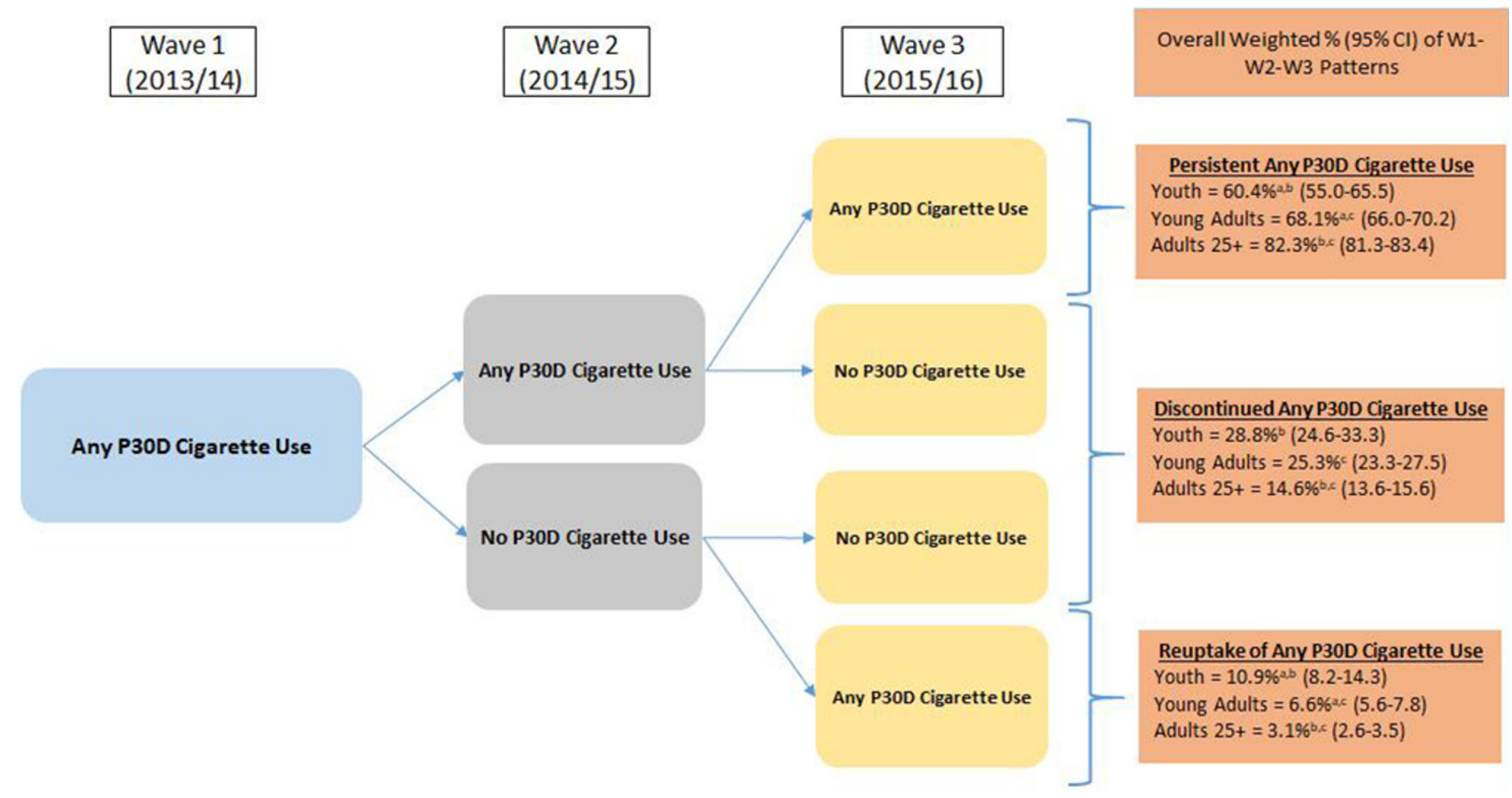

Figure 2 Patterns of W1-W2-W3 persistent any P30D cigarette use, discontinued any P30D cigarette use and reuptake of any P30D cigarette use among $\mathrm{W} 1$ any P30D cigarette users.

Abbreviations: $\mathrm{W} 1=$ Wave $1 ; \mathrm{W} 2=$ Wave $2 ; \mathrm{W} 3=$ Wave $3 ; \mathrm{P30D}=$ past 30-day; $\mathrm{Cl}=$ confidence interval

W1 any P30D cigarette use weighted percentages (95\% Cl) out of total U.S. population: youth (ages 12-17) $=4.5 \%(4.1-5.0)$; young adults (ages 18-

$24)=28.3 \%(26.8-29.7) ;$ adults $25+$ (ages 25 and older) $=21.3 \%(20.7-22.0)$

Analysis included W1 youth, young adults, and adults 25+ P30D cigarette users with data at all three waves. Respondent age was calculated based on age at W1. W3 longitudinal (all-waves) weights were used to calculate estimates. These rates vary slightly from those reported in Figure 1 or Supplemental Table 1 because this analytic sample in Figure 2 includes only those with data at each of the three waves to examine weighted longitudinal use and non-use pathways.

Any P30D cigarette use was defined as any cigarette use within the past 30 days. Respondents could be missing data on other P30D tobacco product use and still be categorized into the following three groups:

1) Persistent any P30D cigarette use: Defined as exclusive or cigarette polytobacco use at W2 and W3.

2) Discontinued any P30D cigarette use: Defined as any noncigarette tobacco use or no tobacco use at either W2 and W3 or just W3.

3) Reuptake of any P30D cigarette use: Defined as discontinued any cigarette use at W2 and any cigarette use at W3.

adenotes significant difference at $p<0.0167$ (Bonferroni corrected for three comparisons) between youth and young adults

${ }^{b}$ denotes significant difference at $p<0.0167$ (Bonferroni corrected for three comparisons) between youth and adults $25+$

'denotes significant difference at $\mathrm{p}<0.0167$ (Bonferroni corrected for three comparisons) between young adults and adults $25+$

The logit-transformation method was used to calculate the $95 \% \mathrm{Cls}$.

Analyses were run on the W1, W2, and W3 Public Use Files (https://doi.org/10.3886/ICPSR36498.v8).

among $28.8 \%$ (95\% CI 24.6 to 33.3 ) of youth, $25.3 \%(95 \%$ CI 23.3 to 27.5 ) of young adults and $14.6 \%$ (95\% CI 13.6 to 15.6) of adults $25+$; the differences between youth and adults $25+$, and between young adults and adults $25+$ were statistically significant. Reuptake of cigarette use occurred among $10.9 \%$ (95\% CI 8.2 to 14.3 ) of youth, $6.6 \%$ (95\% CI: 5.6 to 7.8 ) of young adults and $3.1 \%(95 \%$ CI 2.6 to 3.5$)$ of adults $25+$ and the differences among all three age groups were statistically significant.

\section{Among P30D exclusive cigarette users and P30D cigarette}

\section{polytobacco users at W1}

As shown in the notes to online supplementary figure 2, among adult $25+$ W1 P30D cigarette users, the majority were exclusive cigarette users $(62.6 \%$ (95\% CI: 61.1 to 64.0$))$ compared with cigarette polytobacco users (37.4\% (95\% CI 36.0 to 38.9$)$ ). In contrast, among W1 P30D youth and young adult cigarette users, more were cigarette polytobacco users $(57.1 \%$ (95\% CI 52.1 to 61.9$)$ of youth and $65.2 \%$ (95\% CI 63.0 to 67.4$)$ of young adults). Only $42.9 \%$ (95\% CI 38.1 to 47.9 ) of youth and
$34.8 \%$ (95\% CI 32.6 to 37.0 ) of young adults W1 P30D smokers were exclusive cigarette users. Aim 3 examined 25 possible W1W2-W3 pathways across five mutually exclusive use categories (online supplementary figure 2) among W1 exclusive cigarette users (online supplementary table 1a) and W1 cigarette polytobacco users (online supplementary table $1 \mathrm{~b}$ ). Described below are aggregated pathways from online supplementary table $1 \mathrm{a}, \mathrm{b}$ that estimate broad behavioural transitions such as persistent use, tobacco cessation and relapse in these two W1 user groups (table 1).

Among youth. As shown in table 1, 14.2\% (95\% CI 9.6 to 20.4) of W1 exclusive cigarette users persisted as exclusive users across all three waves, compared with $42.7 \%$ (95\% CI 35.5 to 50.2 ) of W1 cigarette polytobacco users who persisted as cigarette polytobacco users across all three waves. Complementing this finding, $38.8 \%$ (95\% CI 31.8 to 46.3 ) of W1 exclusive cigarette users transitioned to some form of polytobacco use by W3, in contrast to $10.7 \%$ (95\% CI 7.2 to 15.4 ) of W1 cigarette polytobacco users transitioning to exclusive cigarette use by W3. 


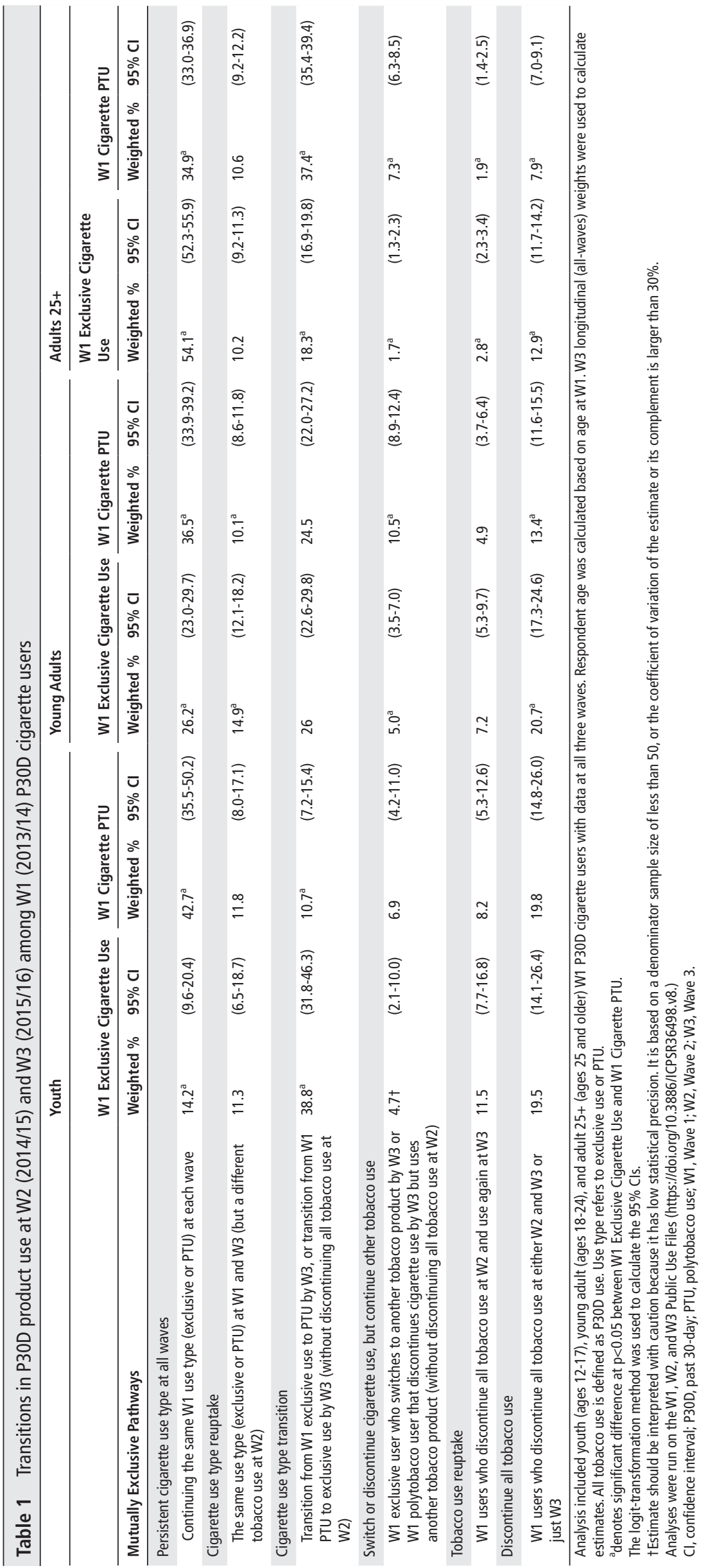


Among young adults. As shown in table 1, 26.2\% (95\% CI 23.0 to 29.7 ) of W1 exclusive cigarette users persisted as exclusive users across all three waves, compared with 36.5\% (95\% CI 33.9 to 39.2 ) of cigarette polytobacco users who persisted as cigarette polytobacco users across all three waves. In addition, there were differences in other pathways such as cigarette use type reuptake and switching or discontinuing cigarette use without quitting tobacco. For instance, $14.9 \%$ (95\% CI 12.1 to 18.2) of W1 exclusive cigarette users stopped exclusive cigarette use at W2 and took it up again at W3, without quitting tobacco entirely, compared with $10.1 \%$ (95\% CI 8.6 to 11.8 ) of W1 cigarette polytobacco users who stopped cigarette polytobacco use at W2 but took it up again at W3, without quitting tobacco entirely. Additionally, while 5.0\% (95\% CI 3.5 to 7.0 ) of W1 exclusive cigarette users discontinued cigarettes while still using tobacco, compared with $10.5 \%$ (95\% CI 8.9 to 12.4 ) of W1 cigarette polytobacco users who discontinued cigarettes while still using tobacco. Finally, 20.7\% (95\% CI 17.3 to 24.6 ) of W1 exclusive cigarette users discontinued all tobacco use by Wave 3 , compared with $13.4 \%$ (95\% CI 11.6 to 15.5 ) of W1 cigarette polytobacco users.

Among adults 25+. As shown in table 1, 54.1\% (95\% CI 52.3 to 55.9) of W1 exclusive cigarette users persisted as exclusive users across all three waves, compared with 34.9\% (95\% CI 33.0 to 36.9 ) of W1 cigarette polytobacco users who persisted as cigarette polytobacco users across all three waves. Additionally, $18.3 \%$ (95\% CI 16.9 to 19.8 ) of W1 exclusive cigarette users transitioned from W1 exclusive use to polytobacco use by W3, compared with $37.4 \%$ (95\% CI 35.4 to 39.4 ) of W1 cigarette polytobacco users who transitioned to exclusive use by W3. Almost 13\% (12.9\% (95\% CI 11.7 to 14.2$)$ ) of W1

\section{What this paper adds}

- This study adds a three-wave examination of cigarette use rates in the United States across multiple definitions of use for youth, young adults and adults $25+$. Across all three waves, young adults have the highest prevalence rates of past 12-month and past 30-day (P30D) use, compared with youth and adults $25+$.

- Longitudinal pathways indicate that cigarette use is persistent over time, with more than $80 \%$ of W1 P30D adult $25+$ cigarette smokers, almost $70 \%$ of W1 P30D young adult cigarette smokers and about $60 \%$ of W1 P30D youth cigarette smokers using cigarettes at all three waves. Since cigarettes have been identified as the most harmful tobacco product on the continuum of risk, the persistent use of cigarettes is a risk to public health.

- Consistent with the finding that the majority of W1 P30D cigarette smokers continued to smoke, $15 \%-30 \%$ of P30D smokers discontinued cigarette use between W1 and W3, with youth having the highest rate of discontinuation and adults $25+$ the lowest.

- While almost $40 \%$ of youth cigarette users transitioned from exclusive cigarette use at W1 to cigarette polytobacco use by W3, only $26 \%$ of young adults and $18.3 \%$ of adults $25+$ followed this pathway. Transitions from W1 cigarette polytobacco use to exclusive cigarette use at W3 occurred in only about $10 \%$ of youth, a quarter of young adults and about one-third of adults $25+$. These finding suggests that youth may be more likely to be cigarette polytobacco users, compared with adults $25+$. exclusive cigarette users discontinued all tobacco use by Wave 3, compared with $7.9 \%$ (95\% CI 7.0 to 9.1) of W1 cigarette polytobacco users.

\section{DISCUSSION}

Cigarettes are the most prevalent form of tobacco used in the USA $^{2}$ as well as the most harmful tobacco product. ${ }^{7}$ This combination of higher prevalence and greater harm has significant implications for public health. In cross-sectional analyses based on data from 2013 to 2016 (W1-W3), prevalence of ever (only among youth), P12M and P30D cigarette smoking decreased among youth and young adults but remained relatively constant for adults $25+$ (although statistically significant changes in adult $25+$ ever, P12M and P30D use were detected, they are of small magnitude and may not be meaningful). Likely of greater import for public health, there were statistically significant decreases in daily cigarette use among both young adults and adults $25+$, with the largest decreases occurring between W1 and W2.

The observed decrease in youth cigarette smoking is consistent with other national surveillance studies, ${ }^{8}$ although the rates of P30D cigarette smoking in 2016 are slightly lower among youth in the PATH Study (5.5\% among 15-17-year olds and 1.0\% among 12-14-year olds), compared with those in the 2016 National Youth Tobacco Survey (NYTS) (8.0\% in high school students and $2.2 \%$ in middle school students). ${ }^{8}$ This may be due to differences in the definition of youth, with high school students aged 18 and older included in the NYTS estimate but not in the PATH Study youth estimate, as well as different skip patterns in the data collection instruments. There is also evidence that household-based assessments of tobacco use prevalence, such as the PATH Study, tend to be lower than schoolbased assessments (eg, NYTS). ${ }^{30}$

Across the 3 years observed in this paper, about $60 \%$ of youth W1 P30D cigarette smokers smoked cigarettes in P30D at all three waves, compared with almost $70 \%$ of young adults and $80 \%$ of adults $25+$. Several other longitudinal studies have identified that cigarette smoking is persistent over even longer time periods. ${ }^{31-33}$ For example, over a 15 -year period of the National Longitudinal Survey of Youth 1997, almost 40\% of established smokers continued to smoke cigarettes over time. ${ }^{17}$ In the Coronary Artery Risk Development in Young Adults study, 52.8\% of baseline current smokers continued cigarette smoking over 25 years. ${ }^{34}$ Given ongoing changes in the US tobacco market, where an increasingly diverse mix of tobacco products, including ENDS, cigars and smokeless tobacco products are available, the finding that cigarette use remains persistent highlights the continued importance of monitoring all tobacco products, including cigarette.

Nevertheless, we also found that $15 \%-30 \%$ of W1 P30D cigarette smokers discontinued cigarette use by W3, with youth having the highest rate of discontinuing cigarettes and adults $25+$ having the lowest. Looking across the papers in this supplement issue, the P30D cigarette discontinuation rate was lower than that of other tobacco products, ${ }^{35-37}$ with the exception of smokeless tobacco. ${ }^{38}$ In contrast to their low P30D cigarette discontinuation rate, adults $25+$ had higher rates of discontinuing ENDS and hookah, compared with youth and young adults. $^{35-38}$

Among W1 P30D exclusive cigarette smokers, less than 15\% of youth, a quarter of young adults and more than half of adults $25+$ used only cigarettes across all three waves without using another product. We also found that among W1 cigarette polytobacco users in all age groups, the most common transition among 
those examined was to continue cigarette polytobacco use at W2 and W3; the second most common transition for young adults and adults $25+$ was a transition back to exclusive cigarette use by W3. This is consistent with studies that have found higher levels of nicotine dependence among polytobacco users, which might lead to continued cigarette use, ${ }^{39}$ and those that have found that cigarette use is persistent over time, as discussed above. The finding that both exclusive and polytobacco cigarette users tend to continue cigarette use further demonstrates the persistence of cigarette use and the pressing need to determine the most effective strategies to help users of all tobacco products, including cigarettes, quit tobacco.

Among youth, the pattern among cigarette polytobacco users was different. After persistent cigarette polytobacco use, the next most common transition among those examined was discontinued use of all tobacco, which likely reflects the more episodic, less stable pattern of youth tobacco use. ${ }^{40}$ However, there are also patterns of youth reuptake of cigarette polytobacco use and transition to exclusive cigarette use, which may indicate that among those youth who continue using tobacco, cigarette use also persists.

Finally, we found that the frequency of transitioning to cigarette polytobacco use among W1 exclusive cigarette smokers differed among age groups. While almost $40 \%$ of youth transitioned from exclusive to cigarette polytobacco use by W3, only $26 \%$ of young adults and $18 \%$ of adults $25+$ followed this pathway. In contrast, transitions from W1 cigarette polytobacco use to exclusive cigarette use at W3 occurred in only about onethirds of adults $25+$, a quarter of young adults and about $10 \%$ of youth. These findings suggest that, compared with adults, youth may be more vulnerable to polytobacco use, which may be due to the appeal of other tobacco products like flavoured products and products that are perceived as at low risk like ENDS. ${ }^{41}$ In addition, it is also not clear if youth use of tobacco products is mere experimentation or if these patterns are indicative of the future long-term use. This report is a resource that provides building blocks to aggregate different pathways to explore a variety of research questions regarding cigarette use.

\section{Limitations}

This study relies on self-reported data, which is subject to recall bias. In addition, discontinued use was defined as no P30D use, without any consideration of intent to quit or duration of cessation, which may have overestimated rates of discontinued use. This study also did not examine transitions in frequency of use, especially transitions to daily use, which may be important to understanding overall patterns of transition. Future studies are needed to examine correlates that predict patterns of transition among exclusive and polytobacco users. Within this journal supplement, Kasza et $a l^{42}{ }^{43}$ and Edwards et $a l^{44}$ examine demographic correlates of initiation, cessation and relapse of cigarette use to further explore predictors of these behavioural outcomes.

\section{Summary and implications}

The persistence of cigarette use among all types of cigarette users (exclusive cigarette users and cigarette polytobacco users) is of particular public health concern since cigarettes have been identified as the most harmful tobacco product. Policy efforts that focus on the continuum of risk, such as the Food and Drug Administration's comprehensive plan for tobacco and nicotine, which is aimed at reducing the harmful effects of tobacco, need to consider a continued focus on cigarettes in addition to new and emerging products such as ENDS.
Contributors KAT and CAS led the conceptual design. KAT drafted the initial manuscript and all authors critically revised it. ES and MJH conducted statistical analysis and all authors contributed to interpretation of the results. All authors approved the work for journal publication and agreed to be accountable for all aspects of the work.

Funding This manuscript is supported with Federal funds from the National Institute on Drug Abuse, National Institutes of Health, and the Center for Tobacco Products, Food and Drug Administration, Department of Health and Human Services, under a contract to Westat (Contract No. HHSN271201100027C).

Disclaimer The findings and conclusions in this report are those of the authors and do not necessarily represent the official position of the US Department of Health and Human Services or any of its affiliated institutions or agencies.

Competing interests WMC reports long-term stock holdings in General Electric Company, 3M Company, and Pfizer Incorporated, unrelated to this manuscript. No financial disclosures were reported by the other authors of this paper.

\section{Patient consent for publication Not required.}

Ethics approval The study was conducted by Westat and approved by the Westat Institutional Review Board. All participants aged 18 and older provided informed consent, with youth participants aged 12-17 providing assent while their parent/ legal guardian provided consent.

Provenance and peer review Not commissioned; internally peer reviewed.

Data availability statement Data from the PATH Study Wave 1 to Wave 3 are available for download as Public Use Files in a public, open access repository (https://www.icpsr.umich.edu/icpsrweb/NAHDAP/studies/36498). Conditions of use are available at the website above.

\section{ORCID iDs}

Kristie A Taylor http://orcid.org/0000-0001-6668-7923

Eva Sharma http://orcid.org/0000-0002-1328-508X

Kathryn C Edwards http://orcid.org/0000-0002-0645-1705

Michael J Halenar http://orcid.org/0000-0002-8703-3811

Karin A Kasza http://orcid.org/0000-0002-4492-098X

Lisa D Gardner http://orcid.org/0000-0003-3340-2818

Maansi Bansal-Travers http://orcid.org/0000-0002-7944-3570

Heather L Kimmel http://orcid.org/0000-0001-8278-0095

Cassandra Stanton http://orcid.org/0000-0001-5329-6261

\section{REFERENCES}

1 Center for Behavioral Health Statistics and Quality. National survey on drug use and health: detailed tables. 2016. Rockville, MD: Substance Abuse and Mental Health Services Administration, 2017.

2 Kasza KA, Ambrose BK, Conway KP, et al. Tobacco-Product use by adults and youths in the United States in 2013 and 2014. N Engl J Med Overseas Ed 2017;376:342-53.

3 Soneji S, Sargent J, Tanski S. Multiple tobacco product use among US adolescents and young adults. Tob Control 2016;25:174-80.

4 Lee $\mathrm{YO}$, Hebert CJ, Nonnemaker JM, et al. Multiple tobacco product use among adults in the United States: cigarettes, cigars, electronic cigarettes, hookah, smokeless tobacco, and snus. Prev Med 2014;62:14-19.

5 El-Toukhy S, Choi K. A Risk-Continuum categorization of product use among US youth tobacco users. NICTOB 2016:18:1596-605.

6 Food and Drug Administration. FDA announces comprehensive regulatory plan to shift trajectory of tobacco-related disease, death [press release]. Silver Spring, MD: Food and Drug Administration, 2017

7 Zeller M, Holman MR, Crosby K. FDA's center for tobacco products: an update on regulatory activities and priorities, 2020.

8 Jamal A, Gentzke A, Hu SS, et al. Tobacco Use Among Middle and High School Students - United States, 2011-2016. MMWR Morb Mortal Wkly Rep 2017;66:597-603.

9 US Department of Health and Human Services. The health consequences of Smoking - 50 years of progress: a report of the surgeon General. Atlanta, GA: US Department of Health and Human Services, Centers for Disease Control and Prevention, National Center for Chronic Disease Prevention and Health Promotion, Office on Smoking and Health, 2014: 943.

10 Jamal A. Current cigarette smoking among adults_United states, 2005-2015. MMWR Morb Mortal Wkly Rep 2016;65.

11 Kahende JW, Malarcher AM, Teplinskaya A, et al. Quit attempt correlates among smokers by race/ethnicity. Int J Environ Res Public Health 2011;8:3871-88.

12 Martell BN, Garrett BE, Caraballo RS. Disparities in Adult Cigarette Smoking - United States, 2002-2005 and 2010-2013. MMWR Morb Mortal Wkly Rep 2016;65:753-8.

13 Reid JL, Hammond D, Boudreau C, et al. Socioeconomic disparities in quit intentions, quit attempts, and smoking abstinence among smokers in four Western countries: findings from the International tobacco control four country survey. Nicotine \& Tobacco Research 2010;12:\$20-33. 
$14 \mathrm{Kim} \mathrm{JE}$, Tsoh JY. Cigarette smoking among socioeconomically disadvantaged young adults in association with food insecurity and other factors. Prev Chronic Dis 2016;13.

15 Buchting FO, Emory KT, Kim Y, et al. Transgender use of cigarettes, cigars, and ecigarettes in a national study. Am J Prev Med 2017;53:e1-7.

16 Matthews AK, Cesario J, Ruiz R, et al. A qualitative study of the barriers to and facilitators of smoking cessation among Lesbian, gay, bisexual, and transgender smokers who are Interested in quitting. LGBT Health 2017;4:24-33.

17 Dutra LM, Glantz SA, Lisha NE, et al. Beyond experimentation: five trajectories of cigarette smoking in a longitudinal sample of youth. PLoS One 2017;12:e0171808.

18 Klein EG, Bernat DH, Lenk KM, et al. Nondaily smoking patterns in young adulthood. Addict Behav 2013;38:2267-72.

19 Saddleson ML, Kozlowski LT, Giovino GA, et al. Assessing 30-day quantity-frequency of U.S. adolescent cigarette smoking as a predictor of adult smoking 14 years later. Drug Alcohol Depend 2016;162:92-8.

20 Lee YO, Hebert CJ, Nonnemaker JM, et al. Youth tobacco product use in the United States. Pediatrics 2015;135:409-15.

21 O'Gara E, Sharma E, Boyle RG, et al. Exploring exclusive and Poly-tobacco use among adult cigarette smokers in Minnesota. Am J Health Behav 2017;41:84-91.

22 Harrell PT, Naqvi SMH, Plunk AD, et al. Patterns of youth tobacco and polytobacco usage: the shift to alternative tobacco products. Am J Drug Alcohol Abuse 2017:43:694-702.

23 Bombard JM, Pederson LL, Nelson DE, et al. Are smokers only using cigarettes? exploring current polytobacco use among an adult population. Addict Behav 2007:32:2411-9.

24 Bombard J, Rock V, Pederson L, et al. Monitoring polytobacco use among adolescents: do cigarette smokers use other forms of tobacco? Nicotine \& Tobacco Res. 2008;10:1581-9.

25 Stanton CA, Sharma E, Seaman EL, et al. Initiation of any tobacco and five tobacco products across 3 years among youth, young adults, and adults in the USA: findings from the PATH Study Waves 1-3 (2013-2016). Tob Control 2020;29:s178-90.

26 Hyland A, Ambrose BK, Conway KP, et al. Design and methods of the population assessment of tobacco and health (path) study. Tob Control 2017;26:371-8.

27 Tourangeau R, Yan T, Sun H, et al. Population assessment of tobacco and health (path) reliability and validity study: selected reliability and validity estimates. Tob Control 2019;28:663-8.

28 McCarthy PJ. Pseudoreplication: further evaluation and applications of the balanced half-sample technique, 1969.

29 Judkins DR. Fay's method for variance estimation. Journal of Official Statistics 1990;6:223.

30 Brener ND, Billy JOG, Grady WR. Assessment of factors affecting the validity of self-reported health-risk behavior among adolescents: evidence from the scientific literature. Journal of Adolescent Health 2003;33:436-57.
31 Augustson E, Marcus S. Use of the Current Population Survey to characterize subpopulations of continued smokers: A national perspective on the "hardcore" smoker phenomenon. Nicotine \& Tobacco Research 2004;6:621-9.

32 Emery S, Gilpin EA, Ake C, et al. Characterizing and identifying "hard-core" smokers: implications for further reducing smoking prevalence. Am J Public Health 2000;90:387.

33 Sorg A, Xu J, Doppalapudi SB, et al. Hardcore smokers in a challenging tobacco control environment: the case of Missouri. Tobacco control 2010;039743.

34 Caraballo RS, Kruger J, Asman K, et al. Relapse among Cigarette Smokers: The CARDIA longitudinal study - 1985-2011. Addict Behav 2014;39:101-6.

35 Stanton CA, Sharma E, Edwards KC, et al. Longitudinal transitions of exclusive and polytobacco electronic nicotine delivery systems (ends) use among youth, young adults, and adults in the USA: findings from the PATH Study Waves 1-3 (2013-2016) Tob Control 2020;29:\$147-54

36 Sharma E, Bansal-Travers M, Edwards KC, et al. Longitudinal pathways of exclusive and polytobacco hookah use among youth, young adults, and adults in the USA: findings from the PATH Study Waves 1-3 (2013-2016). Tob Control 2020;29:s155-62.

37 Edwards KC, Sharma E, Halenar MJ, et al. Longitudinal pathways of exclusive and polytobacco cigar use among youth, young adults, and adults in the USA: findings from the PATH Study Waves 1-3 (2013-2016). Tob Control 2020;29:s163-9.

38 Sharma E, Edwards KC, Halenar MJ, et al. Longitudinal pathways of exclusive and polytobacco smokeless use among youth, young adults, and adults in the USA: findings from the PATH Study Waves 1-3 (2013-2016). Tob Control 2020;29:s170-7.

39 Strong DR, Pearson J, Ehlke S, et al. Indicators of dependence for different types of tobacco product users: descriptive findings from wave 1 (2013-2014) of the population assessment of tobacco and health (path) study. Drug Alcohol Depend 2017; 178:257-66.

40 Orpinas P, Lacy B, Nahapetyan L, et al. Cigarette smoking trajectories from sixth to twelfth grade: associated substance use and high school dropout. NICTOB 2016;18:156-62

41 Mermelstein RJ. Adapting to a changing tobacco landscape: research implications for understanding and reducing youth tobacco use. Am J Prev Med 2014;47:S87-9.

42 Kasza K, Edwards KC, Tang Z, et al. Correlates of tobacco product initiation among youth and adults in the United States: findings from the path study waves 1-3 (20132016). Tob Control 2020;29:s191-202.

43 Kasza K, Edwards KC, Tang Z, et al. Correlates of tobacco product cessation among youth and adults in the United States: findings from the path study waves 1-3 (20132016). Tob Control 2020;29:s203-15.

44 Edwards KC, Kasza K, Tang Z, et al. Correlates of tobacco product relapse among youth and adults in the United States: findings from the path study waves 1-3 (20132016). Tob Control 2020;29:s216-26. 